\title{
Durée d'incubation et effet du stockage des œufs sur le taux d'éclosion dans des lignées de poules sélectionnées sur la consommation alimentaire résiduelle
}

\author{
A Bordas, P Mérat \\ Institut national de la recherche agronomique, laboratoire de Génétique factorielle, \\ centre de Jouy-en-Josas, 78352 Jouy-en-Josas Cedex, France
}

(Reçu le 28 avril 1992, accepté le 15 mars 1993)

\begin{abstract}
Résumé - Deux lignées de poules pondeuses soumises à une sélection divergente sur la consommation alimentaire «résiduelle» $\left(\mathbf{R}^{+}\right.$à consommation élevée, $\mathbf{R}^{-}$à faible consommation) avaient montré antérieurement une différence des taux de fertilité et d'éclosion à l'avantage de $\mathrm{R}^{-}$. Dans le présent travail, elles ont été comparées pour la durée d'incubation (en 1987 et 1988) puis pour la réponse des taux de fertilité et d'éclosion à la durée de stockage des œufs avant la mise en incubation (en 1990). La durée d'incubation a été plus longue pour la lignée $R^{+}$que pour la lignée $R^{-}$, d'environ $9 \mathrm{~h}$ et demie et $8 \mathrm{~h}$ en 1987 et 1988 respectivement. En 1990, on observe au total un désavantage hautement significatif des reproducteurs $\mathrm{R}^{+}$pour le taux d'éclosion, ainsi qu'un effet dépressif de la durée de conservation des œufs sur le taux d'éclosion. Il n'y a pas d'interaction significative lignée $\times$ durée de stockage. Cependant, les données suggèrent une répartition quelque peu différente des causes de pertes à l'éclosion dans les 2 lignées, peut-être influencée par la durée du stockage.
\end{abstract}

poule / consommation alimentaire / fertilité / éclosion

Summary - Duration of incubation and effect of egg storage on hatching rate in lines selected on residual feed consumption. Two lines of laying hens submitted to a divergent selection for residual feed intake ( $R^{+}$with high feed intake, $R^{-}$with low feed intake) had previously shown differences for fertility and hatching rate to the advantage of $R^{-}$. In the present work these lines have been compared for the duration of incubation (in 1987 and 1988), then for their response to the time during which eggs were kept before setting for incubation (in 1990). The duration of incubation was longer for the $R^{+}$line than for $R^{-}$ 
$b y \approx 9.5 \mathrm{~h}$ and $8 \mathrm{~h}$ in 1987 and 1988 respectively. In 1990 on the whole, a highly significant disadvantage was found for the $R^{+}$line in hatchability, as well as a depressing effect of a longer storage of the eggs on hatchability. There was no significant line $\times$ storage duration interaction. However, the data suggest a some what different distribution of the causes of loss at hatching in the 2 lines, possibly influenced by the duration of egg storage.

domestic fowl / feed intake / fertility / hatchability

\section{INTRODUCTION}

Une sélection divergente sur la fraction «résiduelle» de la consommation alimentaire des poules et coqs adultes est poursuivie depuis 1976 à partir d'une population de base Rhode-Island. La réponse directe et certaines réponses liées à cette sélection obtenues après 7 générations dans les lignées divergentes $« \mathrm{R}^{+}$» (consommation élevée) et « $\mathrm{R}^{-}$» (faible consommation) ont été décrites par Bordas et Mérat (1984). Une étude plus complète et récente est présentée par Bordas et al (1992).

Une différence importante entre les 2 lignées pour le taux de fertilité et d'éclosion est apparue dès la $3^{\mathrm{e}}$ génération et s'est maintenue ensuite. Dans la présente note, nous avons voulu comparer entre lignées la durée d'incubation, ainsi que la réponse de chaque lignée à la durée de stockage des œufs avant leur mise en incubation.

\section{MATÉRIEL ET MÉTHODES}

\section{Lignées utilisées et reproduction}

Les lignées $\mathrm{R}^{+}$et $\mathrm{R}^{-}$ont été décrites par Bordas et al (1992). Le critère de sélection $R$, consommation résiduelle, auquel elles étaient soumises de façon divergente, représente l'écart individuel de la consommation alimentaire observée (0) à une valeur de cette consommation prédite à partir d'une équation de régression multiple tenant compte du poids corporel $(P)$, de la variation de poids $(\Delta P)$ et de la masse d'œufs $(E)$ :

$$
R=0-\left(a P^{\alpha}+b \Delta P+c E+d\right)
$$

Ce critère est calculé sur une période de $28 \mathrm{j}$ après le pic de ponte (Bordas, Mérat, 1975). Une variable analogue calculée à partir du poids corporel et de la variation de poids est utilisée chez les coqs. Les résultats reflètent donc l'effet global de la sélection dans les 2 sexes.

La reproduction pedigree dans chaque lignée implique 9 mâles et 45 femelles âgés de 10 à 11 mois avec insémination artificielle de chaque poule placée en cage individuelle tous les $7 \mathrm{j}$. Les reproducteurs sont appariés au hasard, tout en évitant l'accouplement de frères-sœurs ou demi-frères et demi-sœurs. Le pourcentage de ponte (poule/j) contrôlé jusqu'à l'âge de 40 sem est très voisin dans les 2 lignées et varie de 74 à $79 \%$ pour les années considérées. De même, le poids moyen des œufs entre 35 et 40 sem, selon l'année, va de 51,7 à $55,0 \mathrm{~g}$ et 53,0 à $55 \mathrm{~g}$ respectivement dans les lignées $\mathrm{R}^{-}$et $\mathrm{R}^{+}$. 


\section{Dispositif expérimental et observations}

La durée d'incubation a été comparée 2 années successives, en 1987 et 1988. Chaque année, à partir des parents donnant naissance à la génération suivante, une éclosion pedigree supplémentaire correspondant à une collecte des œufs sur 2 sem était effectuée. Les œufs étaient conservés à une température variant entre 12 et $14^{\circ} \mathrm{C}$ avant mise en incubation. L'hygrométrie n'était pas contrôlée de façon précise. Cette éclosion avait lieu respectivement les $16 / 4 / 87$ et $21 / 4 / 88$ (reproducteurs âgés d'environ $1 \mathrm{an}$ ). L'éclosoir était ouvert à différentes heures (tableau I) entre le $20^{\mathrm{e}}$ et le $23^{\mathrm{e}} \mathrm{j}$ après la mise en incubation, et le nombre de poussins éclos à chaque ouverture était noté par lignée. Les œufs bêchés étaient également notés.

Tableau I. Durée d'incubation dans les lignées $\mathrm{R}^{-}$et $\mathrm{R}^{+}$(respectivement à consommation alimentaire « résiduelle» basse et élevée).

\begin{tabular}{|c|c|c|c|c|c|c|}
\hline & & & & & & \\
\hline & $\begin{array}{l}\text { jour } \epsilon \\
\text { d'obse }\end{array}$ & & $N$ & $\%$ & $N$ & $\%$ \\
\hline $\begin{array}{l}1987 \\
\text { J } 20\end{array}$ & $\begin{array}{l}10 \text { h } 30 \\
16 \text { h } 30\end{array}$ & $\begin{array}{c}(0) \\
(+6 \mathrm{~h})\end{array}$ & $\begin{array}{l}0 \\
1\end{array}$ & $\begin{array}{r}\mathbf{0} \\
0,007\end{array}$ & $\begin{array}{l}0 \\
0\end{array}$ & $\begin{array}{l}0 \\
0\end{array}$ \\
\hline J 21 & $\begin{array}{c}9 \text { h } \\
13 \text { h } 30 \\
16 \text { h } 45\end{array}$ & $\begin{array}{c}(22 \mathrm{~h} 30) \\
(27 \mathrm{~h}) \\
(30 \mathrm{~h} 15\end{array}$ & $\begin{array}{r}86 \\
113 \\
119\end{array}$ & $\begin{array}{l}63,7 \\
83,7 \\
88,1\end{array}$ & $\begin{array}{l}18 \\
37 \\
53\end{array}$ & $\begin{array}{l}22,5 \\
46,3 \\
66,3\end{array}$ \\
\hline J 22 & $\begin{array}{r}9 \mathrm{~h} \\
15 \mathrm{~h}\end{array}$ & $\begin{array}{l}(46 \text { h } 30) \\
(52 \text { h } 30)\end{array}$ & $\begin{array}{l}132 \\
135\end{array}$ & $\begin{array}{r}97,8 \\
100,0\end{array}$ & $\begin{array}{l}73 \\
74\end{array}$ & $\begin{array}{l}91,3 \\
92,5\end{array}$ \\
\hline J 23 & $10 \mathrm{~h}$ & $(71$ h 30$)$ & 135 & 100,0 & 80 & 100,0 \\
\hline $\begin{array}{l}1988 \\
\text { J } 20 \\
\text { J } 21\end{array}$ & $\begin{array}{c}15 \mathrm{~h} 30 \\
9 \mathrm{~h} \\
16 \mathrm{~h}\end{array}$ & $\begin{array}{c}(0) \\
(17 \text { h } 30) \\
(24 \text { h } 30)\end{array}$ & $\begin{array}{r}1 \\
121 \\
170\end{array}$ & $\begin{array}{r}0,005 \\
55,3 \\
77,6\end{array}$ & $\begin{array}{r}0 \\
29 \\
48\end{array}$ & $\begin{array}{r}0 \\
27,9 \\
46,2\end{array}$ \\
\hline J 22 & $\begin{array}{r}9 \mathrm{~h} \\
16 \mathrm{~h}\end{array}$ & $\begin{array}{l}(41 \text { h } 30) \\
(48 \text { h } 30)\end{array}$ & $\begin{array}{l}214 \\
217\end{array}$ & $\begin{array}{l}97,7 \\
99,1\end{array}$ & $\begin{array}{r}94 \\
101\end{array}$ & $\begin{array}{l}90,4 \\
97,1\end{array}$ \\
\hline J 23 & $9 \mathrm{~h}$ & $(65$ h 30$)$ & 219 & 100,0 & 104 & 100,0 \\
\hline
\end{tabular}

* Entre parenthèses : heures écoulées après la première observation. $N$ : nombre total d'œuf éclos; \% : pourcentage cumulé.

L'effet de la durée de conservation des œufs avant mise en incubation a été observé en 1990 sur 2 éclosions pedigree successives (les 13/03/90 et 06/04/90), correspondant chacune à la ponte de 3 sem consécutives des reproductrices des lignées $\mathrm{R}^{+}$et $\mathrm{R}^{-}$. Dans chaque lignée, la date de ponte de chaque œuf, d'où découlait sa durée de stockage avant mise à incuber, était enregistrée, et on en déduisait les taux de fertilité et d'éclosion par lignée respectivement pour une durée de conservation comprise entre 0 et $7 \mathrm{j}, 8$ et $14 \mathrm{j}, 15$ et $21 \mathrm{j}$. La fertilité était évaluée à partir des oufs «clairs» au mirage à $5 \mathrm{j}$, avec le risque de compter certains embryons 
morts très tôt avec les œufs infertiles. La mortalité embryonnaire était notée avant et après la mise en éclosoir ( $18^{\mathbf{e}} \mathrm{j}$ d'incubation). Seul le total des 2 éclosions, pour chaque lignée et durée de conservation est présenté, ces 2 éclosions donnant des résultats très voisins.

La température était réglée à $37,8^{\circ} \mathrm{C}$ tout au long de l'incubation et le degré hygrométrique était voisin de 50 et $60 \%$ respectivement en incubateur (jusqu'au $18^{\mathrm{e}} \mathrm{j}$ ) et en éclosoir.

\section{Analyses statistiques}

La durée d'incubation est comparée entre lignées, par un test $t$, en prenant pour centre de chaque classe la demi-somme des durées correspondant aux heures d'ouverture de l'éclosoir entre lesquelles cette classe se situe.

Les effets de la lignée, de la durée de conservation des œufs et l'interaction entre ces 2 facteurs sont testés par une analyse de $\chi^{2}$ (Sokal et Rohlf, 1981).

\section{Résultats}

Le tableau I montre, en 1987 et 1988, les nombres et pourcentages cumulés de poussins éclos aux jours et heures successifs d'observation. Les pourcentages sont rapportés au total des poussins éclos. Le déchet noté à la dernière observation représente les morts en éclosoir; il était respectivement de 6,9 et $12,7 \%$ pour les lignées $\mathrm{R}^{-}$et $\mathrm{R}^{+}$en 1987 , de 13,1 et $26,8 \%$ pour les mêmes lignées en 1988 .

$\mathrm{Du}$ tableau I était déduite la différence moyenne approximative entre lignées pour la durée d'incubation : $9,6 \mathrm{~h}$ et $8,2 \mathrm{~h}$ de moins respectivement en 1987 et 1988 pour la lignée $\mathrm{R}^{-}$que pour la lignée $\mathrm{R}^{+}$. La valeur de $t$ correspondante était de 6,40 en $1987(P<0,001)$ et 6,04 en $1988(P<0,001)$. Les taux d'éclosion (nés/œufs incubés) dans les lots destinés à la reproduction étaient pour 1986 et 1987 respectivement de 62,1 et $64,7 \%$ pour $R^{-}$et 32,4 et 30,1 pour $R^{+}$.

Le tableau II contient les nombres et pourcentages d'œufs «clairs ", d'embryons morts avant et après le $18^{\mathrm{e}} \mathrm{j}$ et de poussins éclos par durée de conservation pour les 2 lignées. L'analyse de $\chi^{2}$ pour les facteurs «lignée» et «durée de conservation» et leur interaction sont données dans le tableau III, pour la proportion de poussins éclos rapportée aux œufs incubés. La signification des effets principaux et l'absence d'intéraction significative se retrouvent également pour les 2 stades de mortalité, dont l'analyse n'est pas présentée dans ce tableau. Il en est de même des œufs «clairs» : pour ces derniers, l'effet «conservation» n'est significatif qu'au seuil $5 \%$, correspondant sans doute à une fraction des «clairs" contenant des morts précoces.

Cependant, en appliquant séparément intralignée un test de $\chi^{2}$ à l'effet «durée de stockage» respectivement pour chaque cause de déchet («clairs», morts avant ou après $18 \mathrm{j}$ ), on observe que dans la lignée $\mathrm{R}^{-}$, cet effet est hautement significatif pour toutes les causes de perte, alors que le même effet n'est significatif que pour la mortalité avant $18 \mathrm{j}$ dans la lignée $\mathrm{R}^{+}$. D'autre part, une analyse de $\chi^{2}$ (non présentée) testant les effets «lignées» et «durée de stockage» en détaillant la classification des œufs non éclos par cause, montre une interaction lignée $\times$ durée de stockage proche de seuil de signification $(P<0,10)$. Ces 2 résultats suggèrent la possibilité d'une répartition quelque peu différente des causes de pertes à l'éclosion 
Tableau II. Fertilité et éclosion des lignées $\mathrm{R}^{+}$et $\mathrm{R}^{-}$selon la durée de stockage des œufs avant incubation.

\begin{tabular}{|c|c|c|c|c|c|c|c|c|}
\hline & \multicolumn{8}{|c|}{ Durée de stockage } \\
\hline & \multicolumn{2}{|c|}{$0-7 j$} & \multicolumn{2}{|c|}{$8-14 j$} & \multicolumn{2}{|c|}{$15-21 j$} & \multicolumn{2}{|c|}{ Total } \\
\hline & $N$ & $\%$ & $N$ & $\%$ & $N$ & $\%$ & $N$ & $\%$ \\
\hline \multicolumn{9}{|l|}{$R^{-}$} \\
\hline Incubés & 407 & & 393 & & 398 & & 1198 & \\
\hline Clairs & 31 & 7,6 & 23 & 5,8 & 49 & 12,3 & 103 & 8,5 \\
\hline Morts avant $18 \mathrm{j}$ & 25 & 6,1 & 34 & 8,6 & 74 & 18,6 & 133 & 11,1 \\
\hline Morts après $18 \mathrm{j}$ & 26 & 6,4 & 48 & 12,2 & 65 & 16,3 & 139 & 11,6 \\
\hline Nés & 325 & 79,8 & 288 & 73,3 & 210 & 52,8 & 823 & 68,7 \\
\hline \multicolumn{9}{|l|}{$R^{+}$} \\
\hline Incubés & 372 & & 375 & & 363 & & 1110 & \\
\hline Clairs & 75 & 20,2 & 67 & 17,9 & 79 & 21,8 & 221 & 19,9 \\
\hline Morts avant $18 \mathrm{j}$ & 32 & 8,6 & 58 & 15,5 & 102 & 28,1 & 192 & 17,3 \\
\hline Morts après $18 \mathrm{j}$ & 62 & 16,7 & 80 & 21,3 & 75 & 20,7 & 217 & 19,5 \\
\hline Nés & 203 & 54,6 & 170 & 45,3 & 107 & 29,5 & 480 & 43,2 \\
\hline \multicolumn{9}{|l|}{ Ensemble } \\
\hline Incubés & 779 & & 768 & & 761 & & 2308 & \\
\hline Clairs & 106 & 13,6 & 90 & 11,7 & 128 & 16,8 & 324 & 14,0 \\
\hline Morts avant $18 \mathrm{j}$ & 57 & 7,3 & 92 & 12,0 & 176 & 23,1 & 325 & 14,1 \\
\hline Morts après $18 \mathrm{j}$ & 88 & 11,3 & 128 & 16,7 & 140 & 18,4 & 356 & 15,4 \\
\hline Nés & 528 & 67,8 & 458 & 59,6 & 317 & 41,7 & 1303 & 56,5 \\
\hline
\end{tabular}

$\mathrm{N}$ : nombre total d'œufs éclos; \% : pourcentage cumulé.

Tableau III. Fertilité et éclosion des lignées $\mathrm{R}^{+}$et $\mathrm{R}^{-}$selon la durée de stockage des œufs : analyse du $\chi^{2}$.

\begin{tabular}{lcc}
\hline Source de variation & $d l$ & $\chi^{2}$ \\
\hline Lignées & 1 & $43,20^{* * *}$ \\
Semaine de stockage & 2 & $32,72^{* * *}$ \\
Interaction & 2 & $1,27 \mathrm{NS}$ \\
\hline
\end{tabular}

${ }^{* * *} P<0,001 ;$ NS : non significatif.

dans les lignées comparées, peut-être en outre selon la durée de stockage avant incubation.

\section{DISCUSSION ET CONCLUSION}

L'allongement de la durée moyenne d'incubation dans la lignée $\mathrm{R}^{+}$forte consommatrice par comparaison à la lignée $\mathrm{R}^{-}$peut rendre compte d'une partie de la différence de taux d'éclosion entre ces 2 lignées dans les conditions ordinaires d'incubation où les poussins nés sont recueillis après une ouverture unique de l'éclosoir (le matin du $22^{\mathbf{e}} \mathrm{j}$ d'incubation dans toute la présente expérience de sélection). 
Cependant, cette explication ne peut être que très partielle, car la différence entre les lignées $\mathrm{R}^{+}$et $\mathrm{R}^{-}$inclut aussi la mortalité embryonnaire précoce, ainsi que le déchet final restant après éclosion (tableau II et références déjà citées récapitulant les données des générations antérieures).

Nous n'avons pas pour l'instant d'interprétation de la différence constatée dans le rythme de développement embryonnaire de nos 2 lignées, mais il paraît vraisemblable qu'il s'agit d'une réponse liée plutôt que d'une dérive génétique aléatoire au cours des générations. On peut mentionner par ailleurs un effet analogue associé à une autre expérience de sélection : une lignée sélectionnée pour donner des poulets «maigres » montre une durée d'incubation inférieure à celle sélectionnée en sens contraire pour donner des poulets "gras " (Bednarczyk et al, 1984). On peut se demander si une différence de thermogenèse entre embryons des lignées $\mathrm{R}^{+}$et $\mathrm{R}^{-}$peut jouer un rôle. Une température corporelle légèrement inférieure chez les adultes de la lignée $\mathrm{R}^{-}$a été notée (Bordas et al, 1992).

La durée de conservation des œufs avant incubation fait apparaître d'autre part un effet dépressif prévisible sur la survie embryonnaire à tous les stades, plus marqué, semble-t-il, avant le $18^{\mathrm{e}} \mathrm{j}$ d'incubation. Néanmoins, la réponse des 2 lignées $\mathrm{R}^{+}$et $\mathrm{R}^{-}$ne présente pas d'interaction significative avec ce facteur : la lignée $\mathrm{R}^{+}$ a un taux de mortalité embryonnaire et d'œufs «clairs» plus élevé que $\mathrm{R}^{-}$, quelle que soit la durée de stockage des œufs.

La répercussion possible d'une divergence des lignées pour des caractères de qualité des œufs sur leur taux d'éclosion respectif sera examinée dans un autre article. Ainsi, la perte de poids des œufs au cours de leur conservation n'a pas été trouvée significativement différente dans les lignées $\mathrm{R}^{+}$et $\mathrm{R}^{-}$en 1983 (données non publiées) mais la différence de taux d'éclosion entre ces lignées à cette époque était moins importante que maintenant. Ce point devrait être vérifié de nouveau.

\section{REMERCIEMENTS}

Ce travail a bénéficié d'une aide financière de l'AIP 91/4765 intitulée «Variabilité génétique de la viabilité embryonnaire».

\section{RÉFÉRENCES}

Bednarczyk MF, Simon J, Ferré R, Leclercq B (1984) Effet de la lumière sur le développement embryonnaire dans 2 lignées de poulets rendus maigres ou gras par sélection. Reprod Nutr Dév 24, 235-238

Bordas A, Mérat P (1975) Enregistrement sur une courte période de la consommation d'aliment chez la poule pondeuse pour l'étude génétique de l'efficacité alimentaire. Ann Génét Sél Anim 7, 331-334

Bordas A, Mérat P (1984) Réponses liées dans une expérience de sélection sur la consommation alimentaire «résiduelle» de coqs et poules Rhode-Island. $17^{\mathrm{e}}$ Congrès mondial d'aviculture, Helsinki, 106-108

Bordas A, Tixier-Boichard M, Mérat P (1992) Direct and correlated responses to divergent selection for residual food intake in Rhode-Island red laying hens. $\mathrm{Br}$ Poult Sci 33, 741-754

Sokal RR, Rohlf FJ (1981) Biometry, 2d ed Freeman \& $C^{\circ}$, San Francisco, 747-764 